\title{
Zur Behandlung der Syphilis
}

mit subcutanen Sublimatinjectionen.

Dr. Anatol Schmidt, klinischem Professor in Warschau.

Im Winter des vergangenen Jahres (1869) haben wir an unserer Abtheilung für Syphilis und Hautkrankheiten beim Ujazdow'schen Militärhospital in Warschau Versuche angestellt, Kranke mit Sublimatinjectionen nach Dr. Lewin's Methode zu behandeln. Vor Allem haben wir uns zur Aufgabe gestellt, vorurtheilsfrei dabei zu Werke zu gehen, um möglichst richtig die Vorzüge einer solchen Behandlung prüfen zu können.

Da wir über ein Material von mehr als 2000 syphilitischen Kranken jährlich verfügen, so haben wir zum Zweck unserer Beobachtungen Subjecte aussuchen können, welche allen bei dieser Behandlung erforderlichen Bedingungen am meisten zu entsprechen schienen. Wir können freilich mit der beschränkten Zahl unserer Experimente Lewin's enormer Statistik nicht entgegentreten, wir glauben jedoch, dass selbst diese geringe Anzahl von Beobachtungen uns zu Schlüssen über den Werth der Sublimatinjectionen berechtigt.

Wir haben vorzüglich Kranke gewählt, welche zu beständig in Warschau cantonnirenden Regimentern gehörten, damit die Patienten nach beendigter Cur unserer ferneren Beobachtung nicht entzogen werden möchten, wenn sie von Recidiven befallen werden sollten. Ausserdem wurde die grösste Aufmerksamkeit der Anamnese geschenkt, und alle in dieser Hinsicht żweifelhaften Fälle aus der angegebenen Behandlung ausgeschlossen.

Die Zahl unserer vorläufigen Beobachtungen beläuft sich auf 37 Fälle. Nach Auspitz's Vorschlag, welchen wir für den 
rationellsten halten, theilten wir die Formen in acute und chronische. Um jedoch den Grad von Wichtigkeit einzelner Species für diese Methode zu verwerthen, ordneten wir unsere Fälle nach den bis jetzt gebräuchlichen Species. Davon waren:

1. Indurirtes Geschwür . . . . . . . . . . . . 5 Fälle.

2. Indurirte Papel . . . . . . . . . . . . . . . 2 " . .

3. Primäre Induration und Leistenbubo . . . . . . . . 7 n

4. Indurirtes Geschwür, Bubo und Roseola . . . . . . . 3 "

5. Bubo ohne Induratio primaria . . . . . . . . . . 5."

6. Doppelseitiger Bubo gleichzeitig mit einer primären Penisinduration ................. $\frac{2}{\text { Summa } 24 \text { Fälle. }}$

7. Anuscondylome ................... 2 ". .

8. Angina catarrhosa, condylomata et roseola ....... 2 "

9. Angina ulcerosa . ............... 4 "

10. Angina ulcerosa et condylomata ........... . . 2",

11. Angina ulcerosa et Syphilis cutanea papulo-lenticularis . . 2 2 "

12. Dolores osteocopi, làryngitis et aphonia chronica - Syphilis

cutanea gummosa (S. tarda) . . . . . . . . . $\frac{1}{.} \quad \frac{1}{\text { Summa }} 13$ Fälle

im Ganzen 37 n

Die ausgewählten Kranken waren junge Soldaten, der jüngste 20, der älteste 35 Jahre alt. Der Constitution nach gehörten 18 żu robusten, früher nicht krank gewesenen Subjecten; von diesen 18 Fällen betrafen 16 die acuten, 2 die chronischen Formen. Ferner waren 13 Individuen, nämlich 6 acute und 7 chronische Fälle, schwächlicher Constitution, welche jedoch ausser Scrophulose im Kindesalter an keiner wichtigen Krankheit vor der syphilitischen Ansteckung gelitten haben. Endlich 6 Patienten waren deutlich durch den chronischen Verlauf und die angewendeten antisyphilitischen Methoden an Kräften herabgekommen. Zwei davon haben vor ein paar Jahren das typhoide Fieber durchgemacht, einer litt an chronischer Lungentuberculose. Wir würden uns nicht entschlossen haben, die Kranken dieser letzten Kategorie einer Sublimatcur zu unterwerfen; wenn nicht Lewin uns dazu ermuthigte, indem er diese Behandlung (Injectionsmethode) bei sonst angegriffenen und brustschwachen Subjecten am geeignetsten findet.

Was die Incubation anbelangt, so schwankte sie bei acuten Fällen zwischen dem 10. und 25. Tage, bei chronischen zwischen 
6 Wochen und 6 Monaten. Der unter Nr. 12 angeführte Fall von Syphilis tarda gehörte einem Kranken an, welcher, seiner Aussage nach, 7 Jahre an recidivirenden Formen der Syphilis behandelt worden ist.

Die acuten 24 Fäle betrafen Patienten, welche zum ersten Mal angesteckt wurden, und obgleich 7 davon mit schon zerfallenden primären Genitalindurationen in's Hospital traten, gebrauchten sie zu Hause doch kein mercurielles Präparat.

Der Vergleichung halber wurden andere 37 Kranke unter. ganz ähnlichen Bedingungen ausgesucht; die Behandlung dieser letztern wurde mit verschiedenen mercuriellen Präparaten nach den sonst üblichen antisyphilitischen Methoden eingeleitet.

Die Injectionen wurden vom assistirenden Arzte Dr. Putilowski unter unserer Controle ausgeführt, dabei sorgfältig die von Lewin angegebenen Bedingungen bei der Operation beobachtet. Täglich wurde bei der Morgenvisite eine Injection gemacht, wiederholte Einspritzungen baben wir nicht versucht, und wollen solche bei einer nächsten Reihe von Experimenten in Anwendung bringen.

Zur Injection wurde eine gewöhnliche graduirte kleine Spritze benutzt; das Instrument war mit stählerner Canüle versehen. Die Sublimatflüssigkeit hatte die drei von $\mathrm{Lew}$ in angegebenen Concentrationsgrade.

Nach unserer Berechnung wurde $z u$ einer schwachen Injection gr. $1 / 8$, zur mittleren gr. $1 / 6$, zur starken gr. $1 / 4$ gebraucht.

Unter solchen Verhältnissen wurde von den 37 Fällen nur bei 21 , namentlich 14 acuten und 7 chronischen, die Behandlung mittelst Injectionen zu Ende geführt. 10. Kranke haben wegen Schmerzen die Anwendung dieser Methode gänzlich verweigert. Bei 6 Kranken, obgleich sie ausschliesslich endermatisch mit Sublimatinjectionen behandelt wurden, haben wir uns genöthigt gesehen, auf gewisse Zeit die Einspritzungen auszusetzen und locale Mittel anzuwenden. Endlich haben 3 acute und 4 chronische Fälle nachträglich mittelst anderer Methoden behandelt werden müssen. 
Von den erfolgreich ohne Unterbrechung ausgeführten Injectionen kamen 3 Fälle auf die schwache, 16 auf die mittlere und 2 auf die starke Sublimatsolution.

Als Nachbehandlung wurde in 3 Fällen innerlich Kali jodatum, bei 1 Sublimat und bei 2 äusserlicher Gebrauch der mercuriellen Salbe verordnet. Die innere Sublimatanwendung betraf einen acuten, die übrigen 4 Fälle chronische Kranke.

Das Maximum der Injectionen belief sich auf 18 Tage, was für die schwache Lösung $2 \frac{1}{4} \mathrm{gr}$, für die mittlere circa $3 \mathrm{gr}$. ausmacht. Bei starker Lösung stiegen wir nur in einem Fall über 15 Injectionen, wo 18 . Einspritzungen gemacht wurden, circa $4 \frac{1}{2}$ gr. Sublimat.

Die Diät wurde individuellen Umständen entsprechend angeordnet; meistens bekamen die Kranken die mittlere Portion (Suppe, Gèmüse, Weissbrod), Wein wurde gänzlich untersagt, Rauchen wurde den daran Gewohnten nicht verboten.

Da unsere Beobachtungen im Winter angestellt wurden, so verblieben die Kranken beständig in einer Temperatur von circa $+18^{\circ} \mathrm{R}$.

Eine Vorbereitungscur wurde nicht verordnet. Einmal wöchentlich gebrauchten die Kranken, der Reinlichkeit wegen, ein Schwitzbad, was bei uns nach volksthümlichem Gebrauch selbst für Gesunde ein Bedürfniss ist.

Unter solchen Bedingungen verliefen die syphilitischen Formen bei dieser Behandlung verschieden. Am schnellsten schwanden die leichten Formen, sowohl primären als recidiven Ursprungs. Ein frisches Geschwür, katarrhalische Angina und leichte Kondylome nebst Roseola schwanden nach Anwendung von 10-12 mittleren Einspritzungen.

Was die primären Genitalgeschwüre anbelangt, so bemerkten wir, dass sie ebenso wie die Indurationen ohne Geschwürsbildung verliefen. Das Geschwür reinigte sich bald, heilte schnell, die Induration dagegen löste sich nur allmälig auf. Dies darf jedoch nicht auffallend erscheinen, da auch bei den sonstigen mercuriellen Methoden das Geschwür im Verhältniss zur Indu- 
ration eine secundäre Rolle spielt. Gegen die primären Indurationen brauchten wir 12-18 bald schwacher, bald mittlerer Injectionen, in einem Falle musste zur Nachcur eine innere Sublimatcur eingeleitet werden.

Schwerer erwies sich der Verlauf ulceröser Anginen und papulöser Hautsyphilis. In solchen Fällen wurden zwar auch nicht mehr als 18 Einspritzungen mittlerer Concentration angewendet, dabei jedoch, was die Halsgeschwüre anbelangt, energische locale Behandlung mittelst wiederholter Cauterisationen und Gargarismata verordnet.

$\mathrm{Zu}$ den schwersten Formen schienen indolente Bubonen zu gehören, und haben wir keinen wesentlichen Unterschied gefunden zwischen solchen, welche eine primäre Ansteckung begleiteten, und anderen, welche als Residua der chronischen Lues nachgeblieben waren. Injectionen in das Gewebe der verhärteten Drüsen haben wir nicht gemacht, obgleich wir Lew in's Besorgnisse in dieser Hinsicht nicht theilen. Zu gleicher Zeit mit den Injectionen wurden in den geschilderten Fällen locale Mittel verordnet, um die Anschwellung möglichst zur Resorption zu bringen. Dessen ungeachtet boten solche Verhärtungen einen hartnäckigen Widerstand. In einigen Fällen erwiesen sich die Einspritzungen ungenügend, wir schritten, wie oben angegeben wurde, zu mercuriellen Einreibungen und innerem Gebrauch von Jodkali. Die Nachcur dauerte so lange, wie bei sonst gewöhnlichem Gebrauch dieser Methoden; es bleibt demnach für diese Fälle unentschieden, welcher Therapie der Erfolg zugeschrieben werden soll.

Was die Complicationen anlangt, so betrafen sie:

1. Schmerzen . . . . . . . 10 Fälle.

2. Fieberhafte Zufälle und Schwäche. 2 n

3. Erysipelas . . . . . . . 1 Fall.

4. Diphtheritische Hautgeschwüre .. 1 n

5. Salivation ........... 1 "

6. Intercostalneuralgie . . . . 1 "

7. Intestinalkatarrh ....... 1 ;

Wir erlauben uns den angegebenen Ergebnissen folgende Bemerkungen hinzuzufügen.

Die Behandlung der Syphilis mit subcutanen Sublimatinjectionen schliesst keineswegs erfolgreiche Resultate aus, 
welche jedoch ganz ebenso jeder anderen mercuriellen Behandlung zukommen, zugleich bedingt sie aber gewisse Complicationen, welche bald ihre Fortsetzung zeitweilig unterbrechen, bald einen weiteren Gebrauch vollständig unmöglich machen. $\mathrm{Zu}$ solchen Complicationen sind wobl vor Allem die Schmerzen zu rechnen, auf welche Lewin sehr wenig Werth legt, welche jedoch, wie unsere Erfahrung zeigt, Vielen so lästig werden, dass sie sich einer weiteren Behandlung nach dieser Methode nicht unterziehen wollen. Von unseren 37 Kranken haben sich 10 wegen der Schmerzen von den Einspritzungen losgesagt. Und doch handelte es sich in unseren Fällen um junge Männer, welche kaum einer übertriebenen Empfindlichkeit beschuldigt werden konnten, ferner waren unsere Patienten Soldaten, von welchen man bei der Behandlung mehr Gehorsam und Geduld erwarten kann, als etwa in der Privatpraxis. In den ersten Tagen ertrugen sie die Injectionen ziemlich geduldig, sie wurden ihnen aber später so lästig, dass sie von dieser Behandlung nichts mehr wissen wollten. Wir wiederholen dabei, dass wir bei unseren Kranken nie mehr als eine Injection täglich verordneten. Den übrigen Complicationen wollen wir keine besondere Wichtigkeit zuschreiben, da solche bei der mercuriellen Therapie nach anderen Methoden gleichfalls vorkommen können.

Der grösste Vorzug der Injectionsmethode wärde hauptsächlich in der Kürze der Behandlung bestehen. Diese würde jedoch nur in dem Falle hoch angeschrieben werden können, wenn keine Recidive nachfolgen würde. Darüber können wir aber vorläufig kein entscheidendes Urtheil abgeben. Zwar bekamen wir nach der Anwendung dieser Methode blos 2 Recidiven zu Gesichte, beide gehören chronischen Kranken an, wir dürfen aber nicht vergessen, dass unsere Experimente kaum ein. halbes Jahr alt sind. 